\title{
Pós-tratamento de lixiviados coagulados por adsorção em resíduos da ostreicultura
}

\author{
Post-treatment of coagulated leachate by adsorption in ostreculture waste
}

\author{
Maurício Alves da Motta Sobrinho'* (1), Patrícia Maria Paulino' (1), \\ Diego Eugênio Bulhões de Oliveira' (i)
}

\section{RESUMO}

Neste trabalho, foi realizado o tratamento do lixiviado de um aterro urbano por meio de uma combinação dos processos de coagulação/floculação e adsorção. O lixiviado empregado foi coletado no aterro de resíduos sólidos urbanos da Muribeca (PE) e coagulado com cal. O objetivo foi avaliar o uso do pó de ostra como adsorvente. Os experimentos de adsorção foram realizados em batelada, utilizando as melhores condições para a ativação e para o processo por intermédio de dois planejamentos experimentais. Em seguida, foram realizados os estudos cinéticos e de equilíbrio e uma avaliação de cada etapa do processo. O tempo de equilíbrio foi de 40 min. As isotermas de adsorção seguiram o modelo de Langmuir e a capacidade máxima de adsorção foi de 45,45 Hazen.L.g' ${ }^{-1}$ A combinação dos processos de tratamento em questão foi bastante eficiente, obtendo reduções de aproximadamente 95\% da demanda bioquímica de oxigênio (DBO), 40\% da demanda química de oxigênio (DQO) e 70\% da cor do lixiviado. Palavras-chave: lixiviado; aterro sanitário urbano; adsorção; coagulação.

\begin{abstract}
In this work the leachate treatment of an urban landfill was carried out through a combination of coagulation/flocculation and adsorption processes. The leachate employed was collected in the municipal solid waste landfill of Muribeca-PE and coagulated with lime. The objective was to evaluate the use of oyster powder as adsorbent. The adsorption experiments were performed in batch using the best conditions for activation and process through two experimental designs. Then, the kinetic and equilibrium studies were carried out as well as an evaluation of each stage of the process. The equilibrium time was 40 min. Adsorption isotherms followed the Langmuir model and the maximum adsorption capacity was 45.45 Hazen.L.g ${ }^{-1}$ The combination of the treatment processes in question was very efficient, achieving a reduction of approximately 95\% DBO, 40\% COD, $70 \%$ of the leachate color.
\end{abstract}

Keywords: urban landfill; adsorption; coagulation.

\section{INTRODUÇÃO}

De acordo com a Associação Brasileira de Limpeza Pública e Resíduos Especiais (ABRELPE, 2017), no Brasil, foram produzidos, em 2017, cerca de 78,4 milhões de toneladas de resíduos sólidos urbanos (RSU), das quais 6,9 milhões não foram sequer coletadas, sendo assim abandonadas em locais inadequados. Já em relação a destinação final adequada dos resíduos coletados (59,1\%), houve uma evolução de 2016 para 2017 de apenas $0,1 \%$. Ressalta-se que, em termos quantitativos, a destinação inadequada cresceu proporcionalmente e que, nesse mesmo período, houve um aumento da disposição em lixões de $0,5 \%$.

No que concerne à composição gravimétrica de diferentes materiais na fração total dos RSU no Brasil, o Plano Nacional de Resíduos Sólidos indica que $51,4 \%$ é de matéria orgânica; $13,5 \%$, de plásticos; 13,1\%, de papel, papelão e Tetra Pak; 2,9\%, de metais; $2,4 \%$, de vidros; e 16,7\%, de outros (BRASIL, 2012). No caso do estado de Pernambuco,

segundo o Plano Estadual de Resíduos Sólidos, a fração de matéria orgânica responde por 56,46\% e os recicláveis, por $25,70 \%$. Esses valores estão bem próximos da média nacional.

$\mathrm{O}$ aterro sanitário continua como o tratamento mais empregado para a disposição de resíduos sólidos urbanos em todo o mundo, o qual gera um efluente altamente complexo e com elevada carga orgânica e toxicidade (LIU et al., 2012). A preferência pela utilização de aterros sanitários deve-se ao fato de esta ser hoje a forma de disposição final mais viável dentro da realidade brasileira, tanto do ponto de vista técnico quanto do econômico. No entanto, o aterro sanitário requer medidas de proteção ambiental local e de suas proximidades, tendo em vista o impacto que este pode causar com a geração de biogás e produtos lixiviados (CASTILHOS JUNIOR, 2006).

Há inúmeros fatores que afetam as características físicas, químicas e biológicas dos lixiviados, por exemplo: idade do aterro, composição e

\section{口-}

IUniversidade Federal de Pernambuco - Recife (PE), Brasil.

*Autor correspondente: mottas@ufpe.br

Recebido: 11/01/2013 - Aceito: 13/05/2019 - Reg. ABES: 109524 
características dos resíduos aterrados (em função das características da população), conteúdo de umidade, precipitação pluviométrica, aspectos climáticos e hidrogeológicos, estações do ano, características do solo, tipo de operação e gerenciamento do aterro, atividade da flora microbiana, além das próprias condições internas do aterro (temperatura, conteúdo de umidade, fase de biodegradação, entre outros) (EL-FADEL et al., 2002; KARGI; PAMUKOGLU, 2003; RENOU et al., 2008a).

Várias tecnologias têm sido empregadas para o tratamento de lixiviados, entre elas podem-se destacar: biodegradação aeróbia ou anaeróbia (HAN; LIU; LI, 2013; ZHANG et al., 2007; XU et al., 2010), lagoas (ROCHA, 2005; MARTIN; JOHNSON, 1995; ROBINSON; GRANTHAM, 1988), Fenton (SINGH; TANG, 2013; ZHANG; CHOI; HUANG, 2005), micro-ondas (CHOU et al., 2013), ultrassom (ROCHEBROCHARD et al., 2013; WANG et al., 2008), microalgas (RICHARDS; MULLINS, 2013; LIN et al., 2007), processos por membranas (MORAVIA; AMARAL; LANGE, 2012; AHMED; LAN, 2012), coagulação (LIU et al., 2012; LI et al., 2010) e assim por diante.

As técnicas mais utilizadas no Brasil e mais comuns para o tratamento de lixiviado, segundo Kurniawan et al. (2006), são as baseadas em processos biológicos, entre as quais destaca-se o sistema de lagoas. Todavia, devido à elevada recalcitrância desse efluente, importantes variações de vazão e de carga reduzem a eficiência do processo e elevam seus custos. A coagulação/floculação é uma técnica de fácil operação e baixo custo (em função do coagulante utilizado), podendo ser empregada para o tratamento de lixiviados estabilizados e antigos nos quais a fração biodegradável é muito pequena (AMOKRANE; COMEL; VERON, 1997; MELLO et al., 2012). Pode também ser utilizada como pré-tratamento (WANG et al., 2009; TATSI et al., 2003; LIU et al., 2012), prévia às biológicas ou outras técnicas físico-químicas. Entre os coagulantes empregados, a literatura cita o sulfato de alumínio $\left(\mathrm{Al}_{2}\left(\mathrm{SO}_{4}\right)_{3}\right)$, o sulfato ferroso $\left(\mathrm{Fe}_{2} \mathrm{SO}_{4}\right)$, o cloreto férrico $\left(\mathrm{FeCl}_{3}\right)$ e o cloreto de polialumínio (PACl) (LIU et al., 2012; LI et al., 2010; MARAÑÓN et al., 2008) ou ainda coagulantes orgânicos (PALA; ERDEN, 2004). O uso da cal (óxido de cálcio) é pouco citado na literatura para o tratamento de lixiviado, entretanto esse produto é de baixo custo e é bastante aplicado no tratamento de água (RENOU et al., 2008b).

No entanto, o uso da coagulação de forma isolada permite apenas remoções moderadas da demanda química de oxigênio (DQO) e da cor, além de gerar um grande volume de lodo e de íons metálicos. Ressalta-se que o processo combinado de coagulação/floculação e adsorção pode minimizar as desvantagens de cada processo. A coagulação/floculação pode remover parte da DQO, reduzindo o consumo do adsorvente. Por outro lado, o carvão ativado em pó (PAC) pode absorver os íons metálicos residuais, assim como a cor, reduzindo o consumo de coagulante e a geração de lodo. Dessa forma, a combinação do processo de coagulação/floculação e adsorção pode ser um método promissor para o tratamento de lixiviados do aterro estabilizados (LI et al., 2010).
Foo e Hameed (2009) elencam 20 artigos que trabalharam com diferentes tipos/percussores de carvão ativado para o tratamento de lixiviado com remoções que variam de 24 a $90 \%$ para a DQO e de 50 a $60 \%$ para a cor. Além desses parâmetros, alguns artigos têm focado a remoção de orgânicos e de amônia. Já Halim et al. (2010) estudaram o uso do carvão e da zeólita em material composto para a remoção de amônia e redução da DQO. O modelo de Langmuir foi o que melhor representou na redução da DQO e da amônia para o carvão e para a zeólita, enquanto para o material composto se seguiu o modelo de Freundlich. Singh et al. (2012) verificaram que não houve diferença entre a adsorção da matéria orgânica entre carvões macro e mesoporosos. Eles obtiveram remoções de $80 \%$ para o carbono orgânico total (COT) e constataram que o material adsorvido preferencialmente nos carvões foi a matéria orgânica de compostos fúlvicos. A aplicabilidade do carvão ativado granular (CAG) na remoção de compostos poluentes foi estudada, em escala de bancada, por Kawahigashi et al. (2014) como pós-tratamento de lixiviado, apresentando eficiência com remoções variando entre 94 e 100\% para cor verdadeira, com valor máximo de $8 \mathrm{uH}$; entre 45 e 76\% para DQO com valor máximo de 167 mg. $\mathrm{L}^{-1}$; e entre 23 e $67 \%$ para COT com valor residual máximo de $93 \mathrm{mg} \cdot \mathrm{L}^{-1}$.

Song e Gao (2013) estudaram a adsorção de cádmio, cobalto, níquel e manganês, presentes em lixiviados sintéticos, em pós de ostra obtendo remoções de 30 a 70\%, em função da massa de adsorvente e do metal. As maiores remoções foram obtidas para o cádmio, com valores entre 55 e $70 \%$.

Kwon et al. (2004) testaram pó de casca de ostra para a remoção de fósforo e verificaram que, em seu estado natural, não conseguiam remover nada, todavia, quando o material foi aquecido, removeu $68 \%$ e, quando pirolisado, removeu até $98 \%$ do fósforo total. Seo et al. (2005) obtiveram remoção de $37,8 \%$ de fósforo, com capacidade de adsorção de $0,833 \mathrm{mg} \cdot \mathrm{g}^{-1}$ e observaram que o modelo de Langmuir foi o que melhor se ajustou. Já Hsu (2009) utilizou pó da casca da ostra para adsorver os íons níquel e cobre em solução aquosa por meio de ensaios em batelada. Ele observou que o modelo de Langmuir foi o que melhor se ajustou aos dados obtidos. A capacidade de adsorção variou de 49,26 a 103,1 mg.g ${ }^{-1}$ para o $\mathrm{Cu}^{2+}$ e entre 48,75 e $94,3 \mathrm{mg} \cdot \mathrm{g}^{-1}$ para o $\mathrm{Ni}^{2+}$

Lim et al. (2012) utilizaram o pó de ostra calcinado para imobilizar metais em solos contaminados com reduções de até $90 \%$, como a que fora atingida para o chumbo. Já Xuan et al. (2010) utilizaram o mesmo composto para a remoção de nutrientes, como meio sortivo "verde" em fossas para evitar contaminação do aquífero. Nessa mesma linha, Cheng et al. (2018) utilizaram o pó de ostra, junto com outros materiais, para a retenção de fósforo em sistemas de tratamento wetlands.

Chaoukia et al. (2017) combinaram os processos de coagulação seguida da adsorção em carvão ativado granular (CAG). Foram obtidos valores de remoção de $77 \%$ para a DQO e de $99,7 \%$ para a cor. 


\section{MATERIAIS E MÉTODOS}

\section{O lixiviado}

O lixiviado bruto foi coletado na caixa medidora de vazão localizada entre a lagoa de decantação e a anaeróbica da Estação de Tratamento de Lixiviados do aterro controlado da Muribeca (Jaboatão dos Guararapes, $\mathrm{PE})$, o qual é considerado um aterro velho.

Para a obtenção do lixiviado coagulado, foi utilizada a cal hidratada (Qualical). Para tanto, inicialmente preparou-se um "leite de cal", por meio da dissolução de $150 \mathrm{~g}$ da cal em $1.000 \mathrm{~mL}$ de água destilada. Em seguida, adicionaram-se $200 \mathrm{~mL}$ dessa solução da cal em $1.800 \mathrm{~mL}$ de lixiviado, compreendendo um volume final de $2.000 \mathrm{~mL}$. Esse volume foi levado a um Jar Test (Quimis ${ }^{\circledR}$, modelo JT-203) utilizando os seguintes parâmetros, otimizados por Paulino (2011): tempo de coagulação de 1 min.; velocidade de coagulação de 115 rpm; tempo de floculação de 25 min.; velocidade de floculação de $36 \mathrm{rpm}$; e tempo de sedimentação de $1 \mathrm{~h}$.

\section{Adsorvente}

$\mathrm{O}$ adsorvente (casca de ostra), coletado no distrito de Tejucupapo (Goiana, PE), foi triturado em moinho de rolos e, em seguida, em moinho de bolas para ser classificado em peneiras da série de Tyler de 20, 60 e 100 Mesh.

Estudos iniciais realizados neste trabalho mostraram a necessidade de um tratamento para as conchas de ostra por não apresentarem uma boa redução de cor, principal parâmetro estudado nesta pesquisa. Foi então realizado um tratamento térmico para aumentar a capacidade do resíduo para o tratamento de lixiviado. Com o uso de um planejamento fatorial, os parâmetros dessa ativação térmica foram otimizados, o que será detalhado posteriormente.

\section{Procedimentos analíticos}

Para a avaliação do processo de tratamento, os parâmetros apresentados na Tabela 1 foram determinados. Essas análises foram realizadas para os lixiviados bruto, coagulado e após o processo de adsorção.

\section{Caracterização do adsorvente}

Para a caracterização do adsorvente, foram realizadas análises da área superficial específica (BET), utilizando Micromeritics ASAP ${ }^{\circledR} 2420$ Accelerated Surface Area and Porosimetry System, do Centro de Tecnologias Estratégicas do Nordeste (CETENE); e de Difração de Raios X em um difratômetro de marca Rigaku, modelo Ultima ${ }^{\circledR}$, operando com tensão de $40 \mathrm{kV}$ e corrente de $20 \mathrm{~mA}$, utilizando radiação K-alfa do cobre.

\section{Planejamento experimental}

Para a otimização do tratamento, inicialmente foi realizada a otimização do adsorvente, verificando as condições ótimas para a sua ativação, seguida de um segundo planejamento para tornar ótimas as condições do processo adsortivo. Cada planejamento foi fatorial completo.

\section{Otimização do adsorvente}

Para a otimização do adsorvente, estudou-se o efeito das variáveis do tratamento térmico (temperatura, tempo de calcinação e granulometria) sobre a capacidade de adsorção da cor pelo pó de ostra calcinado. Realizou-se então um planejamento de $2^{3}$ completo, acrescido de um ponto central. Todos os ensaios experimentais foram realizados em duplicata. Os níveis (mínimo, central e máximo) das variáveis estudadas foram, respectivamente: temperatura da mufla (500; 750; e $\left.1.000^{\circ} \mathrm{C}\right)$, tempo de calcinação $(0,5 ; 1,75 ;$ e $3 \mathrm{~h})$ e faixa granulométrica (20; 60; e 100 Mesh). Esses valores foram fundamentados no estudo de Paulino (2011).

Os experimentos foram realizados aleatoriamente e em duplicata. Em cada erlenmeyer com o adsorvente já pesado (massa do adsorvente $1 \mathrm{~g}$ ), foram adicionados $100 \mathrm{~mL}$ de lixiviado pré-tratado pelo processo de coagulação/floculação. Essa mistura (lixiviado coagulado + adsorvente) foi submetida à agitação, em mesa agitadora orbital Quimis ${ }^{\circledR}$, a $300 \mathrm{rpm}$ durante duas horas. A variável resposta foi a capacidade adsortiva de cor $\left(\mathrm{q}_{\mathrm{t}}\right)$, definida pela Equação 1:

$q_{t}=\frac{\left(C_{i}-C_{f}\right) \cdot V}{m}$

Tabela 1 - Métodos e equipamentos utilizados na caracterização do lixiviado segundo o Standard Methods for the Examination of Water and Wastewater.

\begin{tabular}{|c|c|c|}
\hline Parâmetro & Método & Equipamentos \\
\hline $\mathrm{pH}$ & $\begin{array}{l}\text { Método eletrométrico - } \\
\text { SMEWW } 4500 \text { B }\end{array}$ & $\begin{array}{l}\text { Potenciômetro - DM } \\
22\left(\text { Digimed }^{\oplus}\right)\end{array}$ \\
\hline Cor (Hazen) & $\begin{array}{l}\text { Fotocolorimétrico - } \\
\text { SMEWW } 2120 \text { C }\end{array}$ & $\begin{array}{l}\text { Espectrofotômetro - } \\
\text { Spectroquant Nova } \\
60\left(\text { MERCK }^{\circledR}\right)\end{array}$ \\
\hline $\begin{array}{l}\text { Oxigênio } \\
\text { dissolvido } \\
\left(\mathrm{mg} \mathrm{O}_{2} \cdot L^{-1}\right)\end{array}$ & $\begin{array}{l}\text { Medidor de oxigênio } \\
\text { dissolvido }\end{array}$ & Oxímetro (Digimed $\left.{ }^{\oplus}\right)$ \\
\hline $\begin{array}{l}\text { Condutividade } \\
\left(\mu \mathrm{S} . \mathrm{cm}^{-1}\right)\end{array}$ & $\begin{array}{l}\text { Condutância elétrica } \\
\text { SMEWW } 2510 \text { B }\end{array}$ & $\begin{array}{c}\text { Condutivímetro - DM } \\
32 \text { (Digimed }^{\circledR} \text { ) }\end{array}$ \\
\hline Turbidez (NTU) & $\begin{array}{l}\text { Nefelométrico } \\
\text { SMEWW } 2130 \text { B }\end{array}$ & $\begin{array}{l}\text { Turbidímetro - } \\
\text { turbiquant } 1000 \text { IR } \\
\left(\text { MERCK }^{\oplus}\right)\end{array}$ \\
\hline $\begin{array}{l}\text { Série de sólidos } \\
\left(m g . L^{-1}\right)\end{array}$ & $\begin{array}{c}\text { Gravimétrico } \\
\text { Adaptado do SMEWW } \\
2540-\text { B, } 2540 \text { C, } 2540 \text { D }\end{array}$ & $\begin{array}{l}\text { Estufa Fanen e Mufla } \\
\text { Quimis }^{\oplus} \text { Q318M21 }\end{array}$ \\
\hline $\mathrm{DQO}\left(\mathrm{mg} \mathrm{O}_{2} \mathrm{~L}^{-1}\right)$ & $\begin{array}{c}\text { Titulométrico } \\
\text { (digestão com } \mathrm{K}_{2} \mathrm{Cr}_{2} \mathrm{O}_{7} \text { ) - } \\
\text { SMEWW } 522 \mathrm{C} \mathrm{C}^{-}\end{array}$ & $\begin{array}{l}\text { Termoreator de } \\
\text { DQO para } 8 \text { tubos - } \\
\text { ECO } 8 \text { (Velp") }\end{array}$ \\
\hline $\mathrm{DBO}_{5}\left(\mathrm{mg} \mathrm{O}_{2} \mathrm{~L}^{-1}\right)$ & $\begin{array}{l}\text { Manométrico adaptado } \\
\text { do SMEWW } 5210\end{array}$ & \\
\hline
\end{tabular}

Fonte: APHA; AWWA; WEF (1995). 
Em que:

$C_{\mathrm{i}}=$ cor inicial $(\mathrm{Hz})$;

$C_{\mathrm{f}}=$ cor final $(\mathrm{Hz})$;

$V$ = volume da solução $(\mathrm{L})$;

$m=$ massa de adsorvente $(\mathrm{g})$.

\section{Otimização do processo adsortivo}

Para o segundo planejamento experimental, foram investigados dois fatores que influenciam o processo adsortivo: a quantidade do adsorvente $(\mathrm{m})$ e a velocidade de agitação (A). Dessa forma, foi realizado um planejamento fatorial $2^{2}$ completo em duplicata, adicionado de um ponto central em triplicata. Os níveis (mínimo, central e máximo) das variáveis em estudo nesse planejamento foram: massa (1; 2; e $3 \mathrm{~g})$ e agitação (200; 250; e 300 rpm).

As massas de conchas tratadas, de acordo com o resultado obtido no planejamento fatorial I, foram postas em contato com $100 \mathrm{~mL}$ do lixiviado durante duas horas e a resposta estudada foi a capacidade de adsorção de cor do lixiviado pré-tratado adsorvido por unidade de massa do adsorvente $\left(\mathrm{q}_{\mathrm{t}}\right)$. Para eliminar o efeito da adsorção da cor pelo papel de filtro, foi realizada uma calibração com diferentes concentrações do lixiviado, conforme Paulino (2011).

\section{Estudo do processo adsortivo}

Os experimentos descritos a seguir foram utilizados para realizar estudo cinético do processo adsortivo e estimar parâmetros de equilíbrio, tendo sido realizados em batelada. Nesta etapa, utilizaram-se os melhores resultados obtidos nos dois planejamentos fatoriais.

\section{Estudo cinético}

No estudo cinético, foram usadas $3 \mathrm{~g}$ de massa do adsorvente, tratada termicamente, que foram adicionadas em erlenmeyers que continham $100 \mathrm{~mL}$ do lixiviado pré-tratado sob uma agitação de $300 \mathrm{rpm}$ em temperatura ambiente $\left(30^{\circ} \mathrm{C}\right)$. Os tempos de contato foram de 10, 20, 30, 40, 60, 80, 100 e 120 minutos, nos quais, após a adsorção em cada tempo, a mistura foi filtrada em papel filtro de faixa azul e as cores finais foram determinadas por meio de um Espectrofotômetro Spectroquant ANova $60\left(\operatorname{Merck}^{\circledR}\right)$.

\section{Estudo do equilíbrio}

As amostras de lixiviado foram colocadas sob agitação de $300 \mathrm{rpm}$, durante 60 minutos, $5 \mathrm{~g}$ do adsorvente tratado termicamente em temperatura ambiente $\left(30^{\circ} \mathrm{C}\right)$. Para realizar o estudo de equilíbrio, foi necessário, previamente, obter diferentes concentrações do lixiviado pré-tratado; para isso, foram feitas as seguintes diluições com água destilada (volume do lixiviado/volume da água): 150/0; 140/10; 130/20; 120/30; 110/40; 100/50; 90/60; 80/70; e 70/80, perfazendo 150 mL. Logo após, foram retirados $50 \mathrm{~mL}$ de cada amostra, foi realizada a leitura inicial da cor e os $100 \mathrm{~mL}$ restantes foram submetidos ao processo adsortivo à temperatura ambiente $\left(30^{\circ} \mathrm{C}\right)$. Após a adsorção, o sobrenadante foi filtrado em papel filtro faixa azul, e as concentrações finais da cor do lixiviado foram determinadas através de um espectrofotômetro Spectroquant ANova $60\left(\mathrm{Merck}^{\circledR}\right)$.

\section{RESULTADOS}

\section{Caracterização do adsorvente}

Para a caracterização do adsorvente, foi realizada uma análise da área superficial e volume dos poros (BET) e, por intermédio da difração de raios $\mathrm{X}$, observou-se a composição mineralógica do material.

Na Tabela 2, pode-se observar que o tratamento térmico promoveu aumento significativo - cerca de 50\% - na área superficial BET. O volume poroso aumentou em $74 \%$. Todavia, o diâmetro do poro permaneceu praticamente constante - houve pequena redução de 3\%.

Na Figura 1, é possível verificar que o tratamento favoreceu o aparecimento de poros de menor diâmetro, assim como a redução da quantidade dos poros de maior diâmetro, tendo esse fato proporcionado aumento da área superficial com a manutenção do diâmetro médio dos poros.

A partir das isotermas de adsorção/dessorção (Figura 2) obtidas para o pó de ostra calcinado e tomando como referência a Comunicação de Dados para Sistemas Gás/Solido com especial referência para a determinação da área superficial e porosidade da IUPAC (1985), pôde-se observar que a ostra calcinada apresenta características da isoterma do tipo IV, na qual o ciclo de histerese está associado com a condensação capilar que tem lugar nos mesoporos, limitando a adsorção ao longo de um intervalo de pressão parcial elevada. A parte inicial da isoterma tipo IV é atribuída à monocamada-multicamada de adsorção, uma vez que segue o mesmo perfil que a parte inicial da isoterma tipo II, obtida com os dados sobre a mesma área de uma superfície do adsorvente em uma forma não porosa . Isotermas do tipo IV são comuns em

Tabela 2 - Resultado sumarizado da análise da área específica e do volume dos poros do adsorvente.

\begin{tabular}{|c|c|c|}
\hline Área superficial & In natura & Calcinada \\
\hline Área superficial (BET) $\left(\mathrm{m}^{2} \cdot \mathrm{g}^{-1}\right)$ & 1.3886 & 2.0865 \\
\hline Area superficial (Langmuir) $\left(\mathrm{m}^{2} \cdot \mathrm{g}^{1}\right)$ & 1.8923 & 2.9732 \\
\hline Volume dos poros & In natura & Calcinada \\
\hline $\begin{array}{l}\text { Volume acumulado dos poros na } \\
\text { adsorção com diâmetros entre } 17.000 \\
\text { e } 3.000 .000 \AA\left(\mathrm{cm}^{3} \cdot \mathrm{g}^{-1}\right)\end{array}$ & 0.003982 & 0.006952 \\
\hline Tamanho dos poros & In natura & Calcinada \\
\hline $\begin{array}{l}\text { Diâmetro médio dos poros na } \\
\text { adsorção }\left(4 \mathrm{~V} \cdot \mathrm{A}^{-1}\right)(\mathrm{V})\end{array}$ & 327.004 & 317.118 \\
\hline
\end{tabular}


muitos adsorventes industriais mesoporosos. No ponto B, no início da secção do meio quase linear da isotérmica, é frequentemente tomado para indicar a fase em que a cobertura monocamada está completa e a adsorção de multicamadas, prestes a começar. A histerese se aproxima do tipo H3, na qual são observados agregados de placa de partículas semelhantes, os quais dão origem a poros em forma de fendas.

Através do espectro de difração de raios $\mathrm{X}$ do material in natura (Figura 3A) foi possível verificar que o material apresenta picos característicos da calcita, assim como uma pequena contaminação por quartzo. Essa contaminação pode ser oriunda dos moinhos que promoveram a redução do material.

Após a calcinação (Figura 3B), foram observados picos que caracterizam o material tipicamente como óxido de cálcio. Dessa maneira, a calcinação transformou a calcita em óxido de cálcio.

\section{Planejamento fatorial dos experimentos}

A utilização do planejamento experimental fatorial teve como objetivo realizar um estudo mais abrangente das variáveis independentes, fazendo, de forma mais organizada, uma quantidade mínima de experimentos, nos quais foi utilizado o tempo de duas horas.

\section{Otimização do tratamento do adsorvente}

Nesse primeiro planejamento, foi possível analisar o melhor tratamento térmico para o adsorvente, analisando as variáveis temperatura e tempo de calcinação, bem como a melhor granulometria para o adsorvente, no intuito de obter a máxima adsorção da cor do lixiviado pela concha da ostra.

A partir dos resultados do planejamento fatorial completo, o gráfico de Pareto foi obtido para visualização dos efeitos estimados dos fatores principais. Esse gráfico fornece uma representação gráfica para esses fatores e permite observar a magnitude e a importância de um determinado efeito. Nele (Figura 4), as barras (fatores) que graficamente ultrapassam a linha de significância exercem uma influência estatisticamente significativa sobre o resultado. Dessa forma, foi possível observar que a única variável que influencia de forma significativa o tratamento do adsorvente foi a temperatura.

Os dados dos planejamentos fatoriais também foram submetidos à análise de variância, à análise de regressão e ao teste F. Verificou-se que

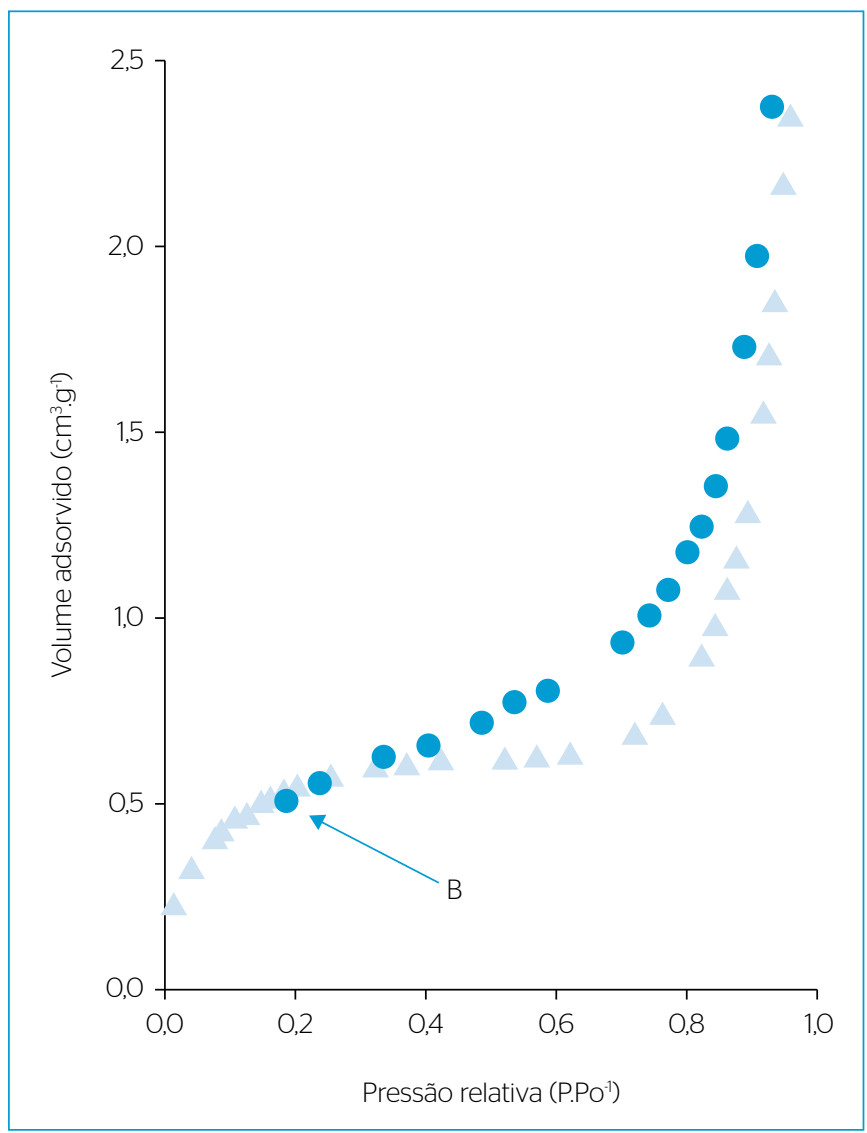

Figura 2 - Isotermas de adsorção $(\Delta)$ e dessorção (o) para a ostra calcinada.
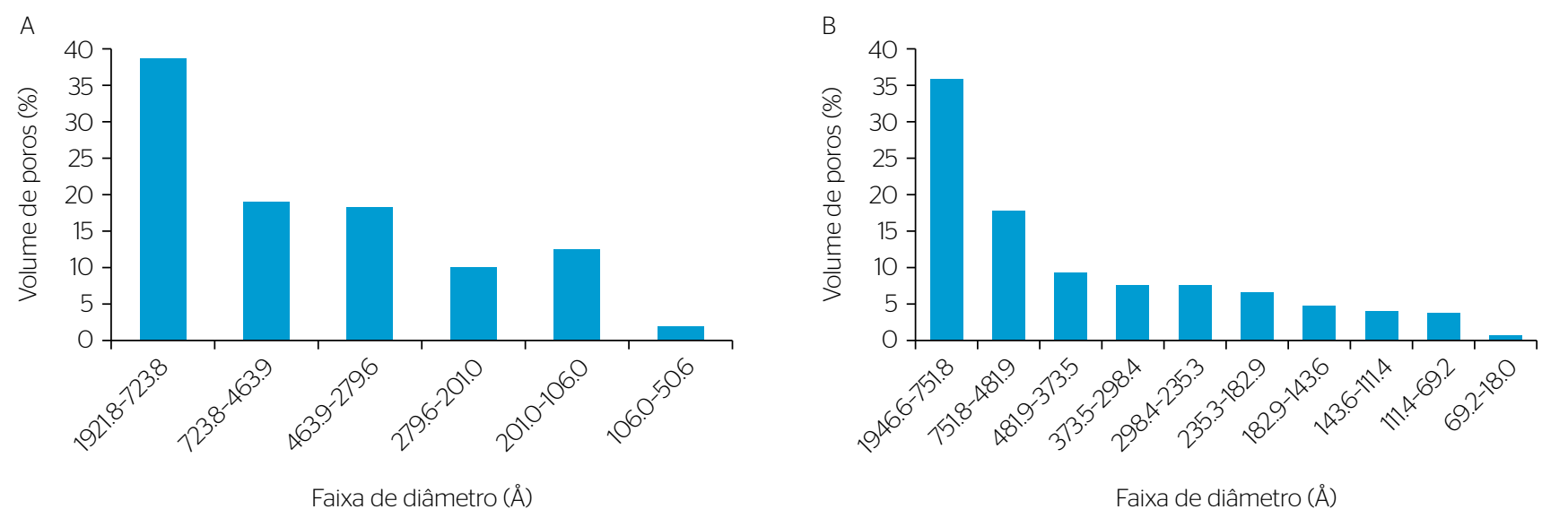

Figura 1 - Distribuição percentual do volume dos poros em função do diâmetro para o pó da ostra in natura (A) e calcinada (B). 
o modelo para otimização do adsorvente apresenta um coeficiente de correlação múltipla $\left(\mathrm{R}^{2}\right)$ igual a 0,73 , as suas variâncias explicáveis (\% Mx explicada e explicável) são iguais a 73,64 e 99,96\%, respectivamente, em que quanto mais próximas de $100 \%$ menos erros estarão sendo adicionados ao modelo, devido à falta de ajuste e aos erros experimentais, e possui ainda uma regressão estatisticamente significativa $\left(\mathrm{F}_{\text {calculado }} / \mathrm{F}_{\text {tabelado }}>1\right.$ ), ao nível de $95 \%$ de confiança de acordo com Barros Neto et al. (2001).

A Figura 5 apresenta as superfícies de resposta construídas com o auxílio do software Statistic ${ }^{\circledR} 5.0$ por meio do uso dos dados obtidos experimentalmente nas melhores condições do adsorvente, conseguidas pelo planejamento fatorial $2^{3}$.

Pela inclinação da superfície de resposta em relação ao eixo da temperatura (Figura 5A), pode-se constatar que esta exerce forte influência no processo. Todavia, em relação à granulometria, as linhas estão praticamente paralelas ao seu eixo, indicando pouca significância desse efeito. Pela Figura 5B, percebeu-se redução da cor quando se utilizou maior faixa granulométrica em Mesh com menor tempo na mufla e maior temperatura, respectivamente.

A partir dos resultados obtidos nessa etapa, os experimentos seguintes foram realizados, utilizando as conchas de ostra tratadas termicamente a $1.000^{\circ} \mathrm{C}$ durante $0,5 \mathrm{~h}$ (30 min), com granulometria de $100 \mathrm{Mesh}$.

\section{Otimização do processo adsortivo}

Nesse planejamento, foi avaliado o efeito das variáveis massa e agitação no processo adsortivo a fim de alcançar a sua otimização. A Figura $6 \mathrm{~A}$ apresenta o gráfico de Pareto obtido com base nos resultados dessa planificação. Infere-se que o efeito é tão significativo na remoção da cor quanto mais à direita da linha vermelha ele estiver. Também são mostrados os efeitos das interações entre duas variáveis. Segundo ainda esta Figura, observa-se que todas as variáveis apresentaram efeito significativo na remoção da cor.

A Figura 6B apresenta a superfície de resposta obtida por intermédio do uso dos dados alcançados experimentalmente, após uma otimização por planejamento fatorial $2^{2}$. Percebe-se que os melhores valores encontrados para a variável resposta foram para os níveis superiores.

Os valores obtidos na análise de variância (ANOVA) para o estudo do processo adsortivo foram calculados mediante a utilização do programa Statistica ${ }^{\circledR}$ 5.0. Uma análise de significância estatística dos valores observados é um fator importante, uma vez que os dados experimentais são utilizados para produzir um modelo empírico, por meio da regressão. O coeficiente de determinação $\mathrm{R}^{2}=0,878$ quantifica a qualidade do ajuste do modelo, pois fornece uma medida da proporção da variação explicada pela equação de regressão em relação à variação total das respostas. $\mathrm{O}$ teste $\mathrm{F}$ apresenta a razão entre o $\mathrm{F}$ calculado e o $\mathrm{F}$ tabelado igual a 6,48. Sempre que essa relação for maior que 1, a regressão é estatisticamente

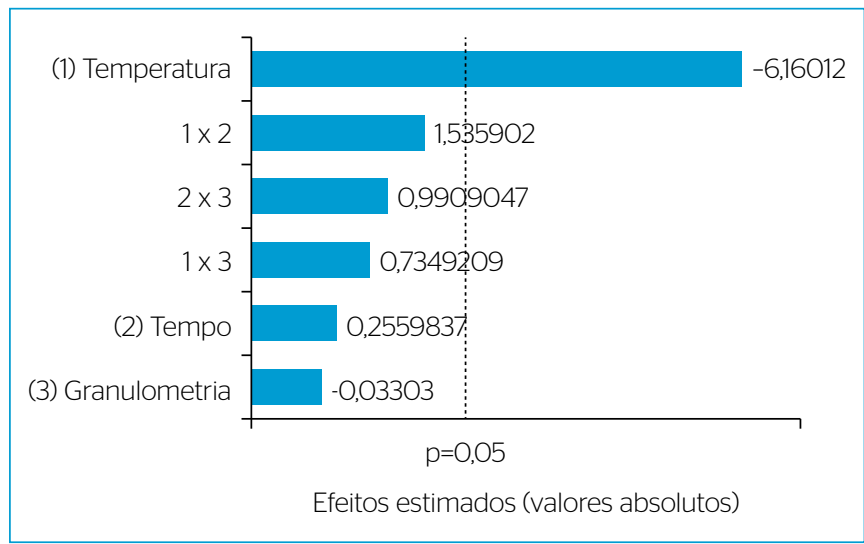

Figura 4 - Gráfico de Pareto referente à análise dos fatores que mais influenciaram na otimização no adsorvente.

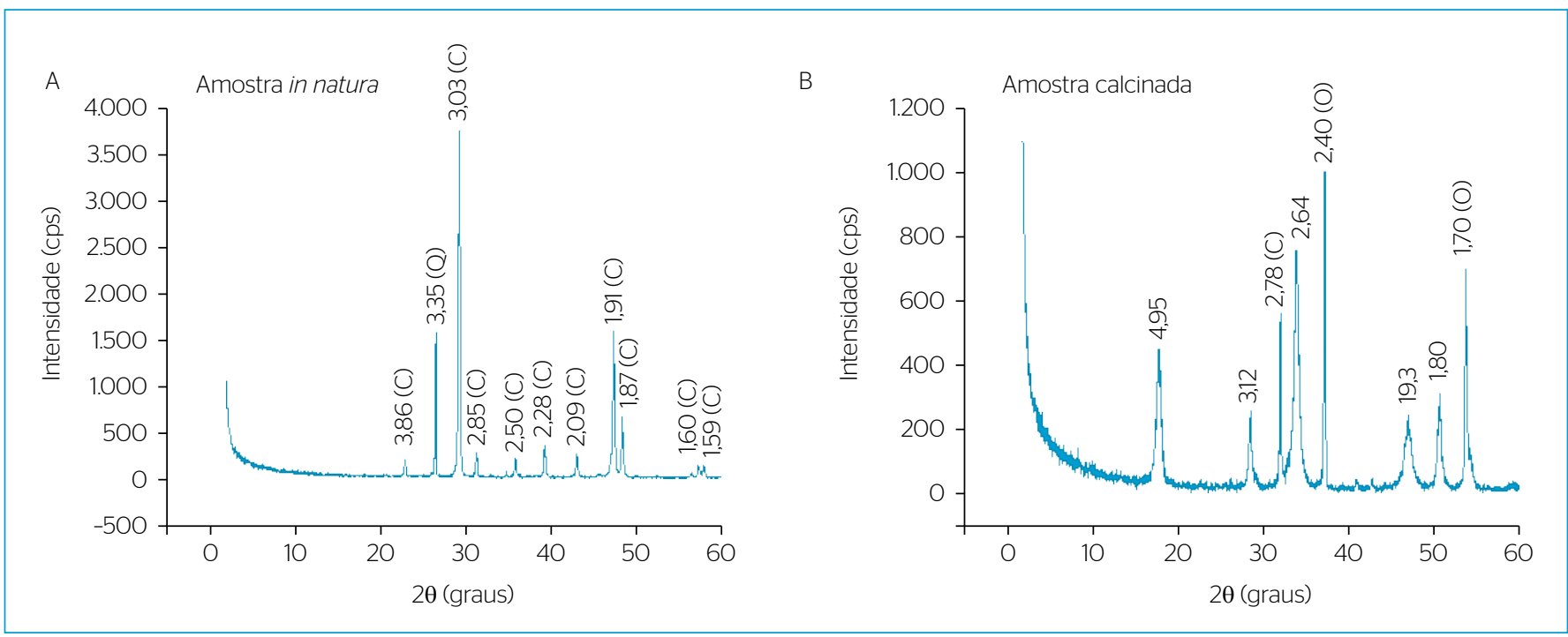

Figura 3 - Espectro de difração de raios $X$ das amostras de pó de ostra in natura (A) e calcinada (B). 
significativa, havendo relação entre as variáveis independentes e dependentes. Para que uma regressão seja não apenas estatisticamente significativa, mas também útil para fins preditivos, o valor da razão deve ser no mínimo maior que 4 (BARROS NETO; SCARMINIO; BURNS, 2001).

A Equação 2 representa o modelo empírico para o sistema experimental analisado por meio da regressão dos dados experimentais do processo adsortivo com relação ao parâmetro resposta cor.

Cor $=-113,74 \mathrm{~A}-278,75 \mathrm{M}-147,50 \mathrm{AxM}$

Em que:

$\mathrm{A}=\operatorname{agitação}(\mathrm{rpm})$;

$\mathrm{M}=$ massa do adsorvente utilizado (g);

AxM= interação entre os dois parâmetros.

\section{Avaliação do processo adsortivo}

Parte-se agora para a avaliação do processo adsortivo na remoção da cor do lixiviado (pré-tratado) pelas conchas da ostra, buscando-se encontrar o tempo de estabilização da adsorção necessários para o estudo de equilíbrio e a capacidade máxima para a remoção de cor.

\section{Estudo cinético de adsorção}

A observação do comportamento cinético da adsorção da cor do lixiviado sobre as conchas de ostra permite a determinação do tempo de equilíbrio, no qual o fluxo de entrada de cor no adsorvato é igual ao da saída. Observou-se (Figura 7) que o processo adsortivo segue uma cinética de adsorção com estabelecimento do equilíbrio próximo de 40 minutos, a partir do qual as variações

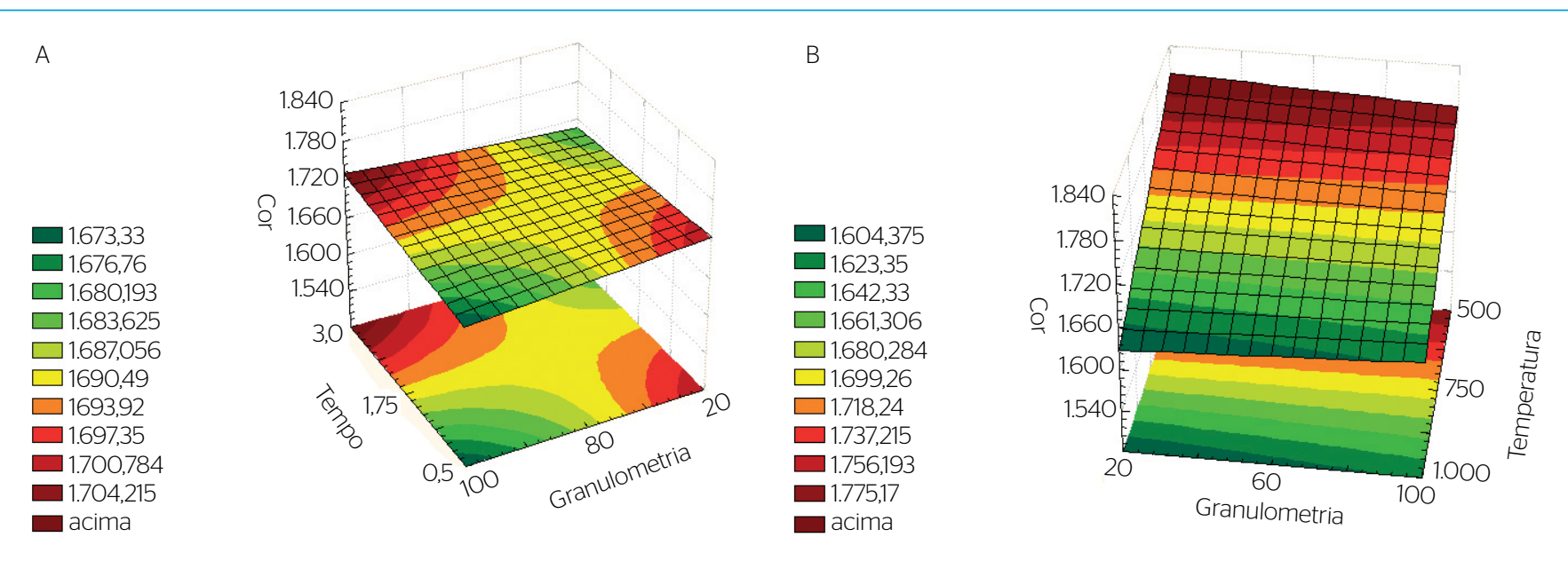

Figura 5 - Superfícies de resposta para a redução da cor do lixiviado pelas variáveis tempo de calcinação e granulometria (A) e granulometria e temperatura (B).
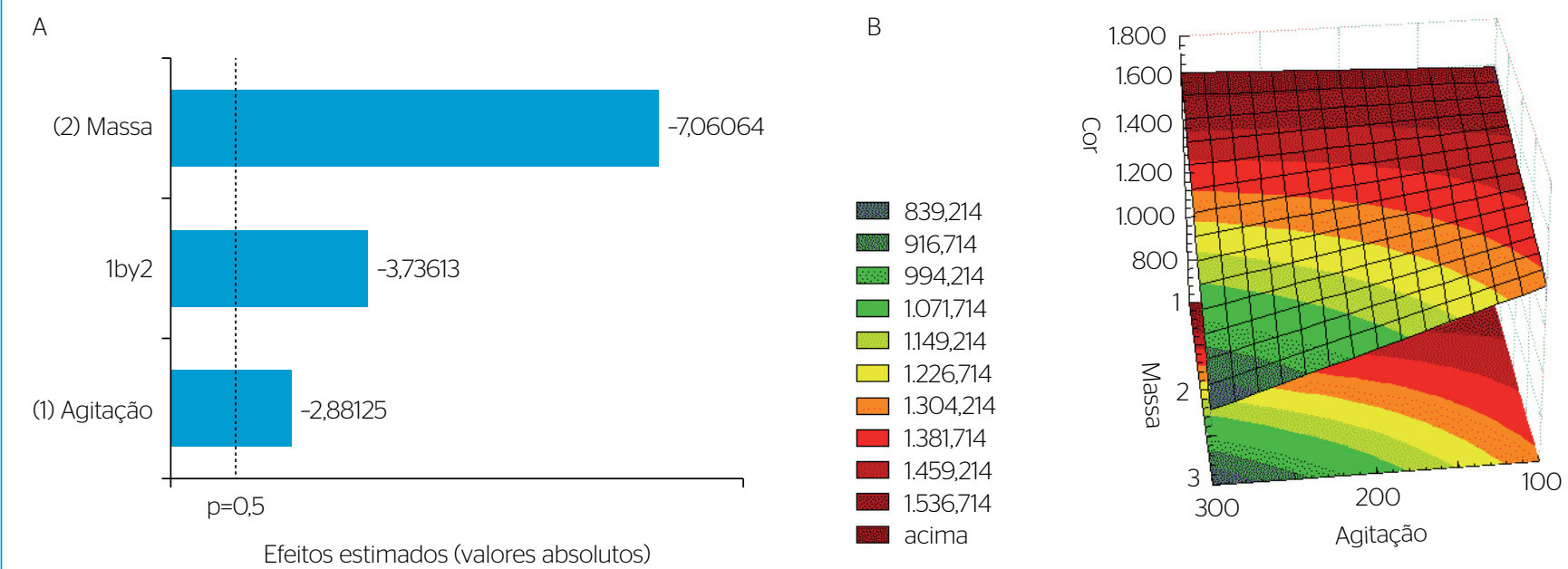

Figura 6 - Gráfico de Pareto referente à análise dos fatores que influenciaram no processo adsortivo (A) e superfície de resposta para a redução da cor do lixiviado pelas variáveis massa e agitação (B). 
de concentração e quantidade adsorvida se tornam insignificantes frente ao gradiente relativo à cor inicial. Para garantir que o sistema se encontra em equilíbrio, os ensaios de equilíbrio foram realizados com 60 minutos.

\section{Estudo do equilíbrio de adsorção}

Nesses ensaios, foram testados os modelos de Langmuir e Freundlich, com o intuito de verificar os ajustes dos dados experimentais da adsorção da cor do lixiviado nas conchas de ostra tratadas. O modelo de Langmuir foi o que mais se ajustou em relação à distribuição dos pontos na curva, com coeficiente de correlação de 0,92 , o que justifica o fato da escolha do modelo (Figura 8). Assim, os valores obtidos de acordo com a equação da reta do modelo de Langmuir linearizado aos dados do experimento foram de 0,006537 L.Hazen ${ }^{-1}$ da constante de equilíbrio da adsorção $\left(\mathrm{K}_{\mathrm{A}}\right)$ e 45,45 Hazen.L.g ${ }^{-1}$ foi sua capacidade máxima de adsorção (saturação na monocamada).

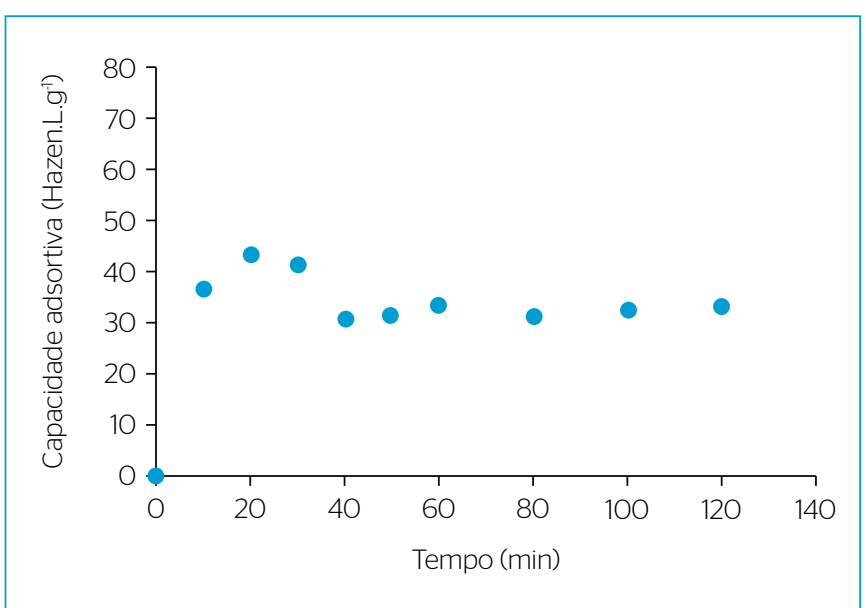

Figura 7 - Evolução cinética da quantidade adsorvida da cor do lixiviado sobre as conchas de ostra com $1 \mathrm{~g}$ de adsorvente $(\mathrm{m}=3 \mathrm{~g}$, agitação $=300 \mathrm{~g}, \mathrm{v}=0,1 \mathrm{~L}$ ).

\section{Avaliação geral do processo}

Com os resultados, esperam-se obter conhecimentos novos sobre o funcionamento do processo de coagulação/floculação, combinado ao de adsorção para degradação da matéria orgânica contida no lixiviado. A Tabela 3 mostra os resultados obtidos pela associação de tratamento composto pelos processos de coagulação/floculação e adsorção.

Tabela 3 - Resultados obtidos no tratamento combinado do lixiviado.

\begin{tabular}{|c|c|c|c|}
\hline Parâmetros & $\begin{array}{l}\text { Lixiviado } \\
\text { bruto }\end{array}$ & $\begin{array}{c}\text { Lixiviado } \\
\text { pré-tratado } \\
\text { pelo processo } \\
\text { de coagulação/ } \\
\text { floculação }\end{array}$ & $\begin{array}{l}\text { Lixiviado } \\
\text { coagulado } \\
\text { tratado pelo } \\
\text { processo de } \\
\text { adsorção }\end{array}$ \\
\hline $\mathrm{DBO}\left(\mathrm{mg} . \mathrm{L}^{-1}\right)$ & 900 & 300 & 50 \\
\hline $\mathrm{DQO}\left(\mathrm{mg} \cdot \mathrm{L}^{-1}\right)$ & 2.878 & 2.453 & 1.762 \\
\hline Condutividade $\left(\mu \mathrm{S} . \mathrm{cm}^{-1}\right)$ & 19.700 & 17.030 & 16.822 \\
\hline $\mathrm{pH}$ & 8,70 & 9,31 & 12,55 \\
\hline $\mathrm{OD}\left(\mathrm{mg}\right.$ de $\left.\mathrm{O}_{2} \mathrm{~L}^{\mathrm{L}^{-1}}\right)$ & 2,09 & 3,75 & 5,48 \\
\hline Cor (HZ - Hazen) & 2.196 & 1.824 & 680 \\
\hline Turbidez (NTU) & 15,45 & 15,44 & 12,19 \\
\hline $\mathrm{ST}\left(\mathrm{mg} . \mathrm{L}^{-1}\right)$ & 8.120 & 7.246 & 7.804 \\
\hline STV $\left(m g . L^{-1}\right)$ & 58 & 136 & 1.055 \\
\hline $\operatorname{STF}\left(\mathrm{mg} \cdot \mathrm{L}^{-1}\right)$ & 8.062 & 7.110 & 6.749 \\
\hline $\mathrm{SST}\left(\mathrm{mg} \cdot \mathrm{L}^{-1}\right)$ & 50 & 205 & 79 \\
\hline $\mathrm{SSV}\left(\mathrm{mg} \cdot \mathrm{L}^{-1}\right)$ & 32 & 105 & 55 \\
\hline $\mathrm{SSF}\left(\mathrm{mg} \cdot \mathrm{L}^{-1}\right)$ & 18 & 100 & 24 \\
\hline SDT (mg. $\left.L^{-1}\right)$ & 8.070 & 7.040 & 7.725 \\
\hline $\mathrm{SDV}\left(\mathrm{mg} \cdot \mathrm{L}^{-1}\right)$ & 26 & 30,5 & 1.000 \\
\hline SDF $\left(m g \cdot L^{-1}\right)$ & 8.044 & 7.009 & 6.725 \\
\hline
\end{tabular}

DBO: demanda bioquímica de oxigênio; DQO: demanda química de oxigênio (DQO); OD: oxigênio dissolvido; ST: sólidos totais; STV: sólidos totais voláteis; STF: sólidos totais fixos; SST: sólidos suspensos; SSV: sólidos suspensos voláteis; SSF: sólidos suspensos fixos; SDT: sólidos dissolvidos; SDV: sólidos dissolvidos voláteis; SDF: sólidos dissolvidos fixos
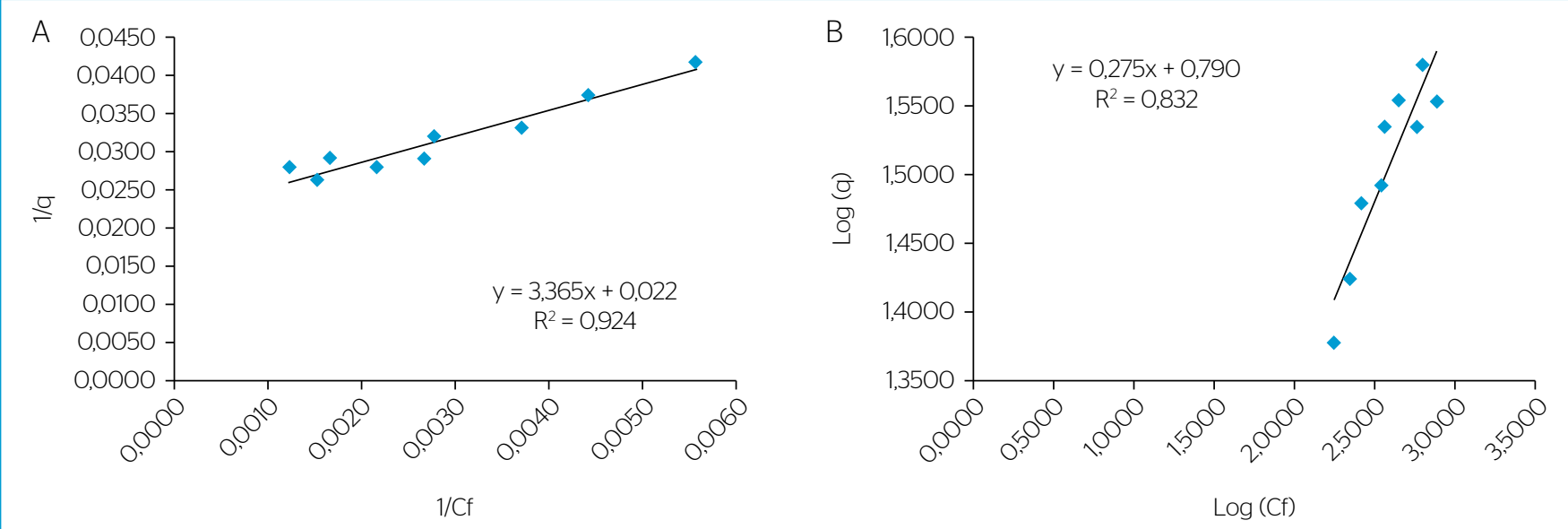

Figura 8 - Ajuste dos resultados experimentais do estudo de equilíbrio na isoterma linearizada de Langmuir (A) e de Freundlich (B). 
Fazendo uso dos resultados apresentados, no geral a combinação dos processos de tratamento em questão foi bastante eficiente em diversos aspectos, obtendo uma redução de aproximadamente $95 \%$ da demanda bioquímica de oxigênio (DBO), 40\% da DQO, 70\% da cor do lixiviado e $21 \%$ da turbidez; em relação aos sólidos, após a combinação de tratamento, verificou-se que cerca de $86 \%$ deles são de origem inorgânica (sólidos fixos) - antes do tratamento essa porcentagem era de $99 \%$ - e o oxigênio dissolvido mostrou melhora de 2,6 vezes a mais após o tratamento.

Em comparação com outros tratamentos alcançados para lixiviados, temos os seguintes resultados: em trabalho realizado por Aragão et al. (2009), no qual foi avaliada a eficiência do processo de coagulação/floculação seguido do de oxidação avançada com reagente de Fenton na degradação do lixiviado produzido pelo lixão da Terra Dura de Aracaju (SE), obteve-se uma redução de 61,5\% de sua cor; já a pesquisa de Rodrigues Filho et al. (2008), que utilizou um processo de oxidação avançada por foto-Fenton no lixiviado do aterro sanitário do Cachimba, na região metropolitana de Curitiba (PR), obteve 59\% de redução da cor. Tendo em vista essas pesquisas citadas e os resultados obtidos neste trabalho, pode-se afirmar que essa combinação de tratamento é bastante eficiente na remoção das substâncias recalcitrantes (cor do lixiviado) e em diversos fatores, tais como: custo, simplicidade no processo, melhores resultados na diminuição dos poluentes potenciais e utilização de resíduos descartados (conchas de ostra) como adsorventes.

\section{CONCLUSÕES}

Por meio de um planejamento experimental fatorial, as conchas de ostra (resíduo da ostreicultura) mostraram que, após pulverizadas a uma granulometria de $100 \mathrm{Mesh}$ e ativadas termicamente a $1.000^{\circ} \mathrm{C}$, durante $30 \mathrm{minu}$ tos, apresentaram boa capacidade de adsorver as substâncias recalcitrantes (cor do lixiviado). Essa ativação resultou em um aumento de, aproximadamente, $50 \%$ na sua área superficial e $74 \%$ no seu volume de poros.

Ainda fazendo uso do planejamento experimental para otimização do processo adsortivo, concluiu-se que as melhores relações entre a cor do lixiviado e a capacidade de adsorção do adsorvente são a granulometria e a ativação térmica das conchas da ostra ditas anteriormente, a quantidade do adsorvente de $3 \mathrm{~g}$ e a velocidade de agitação de $300 \mathrm{rpm}$.

O processo adsortivo alcançou o equilíbrio no tempo de 40 minutos. Os dados experimentais das isotermas de adsorção se ajustam ao modelo da isoterma de Langmuir, com capacidade máxima de adsorção de 45,45 Hazen.L.g ${ }^{-1}$.

Com a combinação dos processos de coagulação/floculação e adsorção, conseguiu-se remoção de quase $70 \%$ da cor do lixiviado, 95\% da DBO, $40 \%$ da DQO e $21 \%$ da turbidez.

\section{REFERÊNCIAS}

AHMED, F.N.; LAN, C.Q. (2012) Treatment of landfill leachate using membrane bioreactors: a review. Desalination, v. 287, p. 41-54. https://doi.org/10.1016/j.desal.2011.12.012

AMOKRANE, A.; COMEL, C.; VERON, J. (1997) Landfill leachate pretreatment by coagulation-flocculation. Water Research, v. 31, n. 11, p. 2775-2782. https://doi.org/10.1016/S0043-1354(97)00147-4

AMERICAN PUBLIC HEALTH ASSOCIATION (APHA); AMERICAN WATER WORKS ASSOCIATION (AWWA); WATER ENVIRONMENT FEDERATION (WEF). (1995) Standard methods for the examination of water and wastewater. 18. ed. Washington, D.C.

ARAGÃO, L.I.N.S.; LIMA, N.S.; LIMA, A.S.; CAVALCANTE, E.B. (2009) Tratamento do chorume pelo processo oxidativo avançado Reagente Fenton. In: ENCONTRO SOBRE APLICAÇÕES AMBIENTAIS DE PROCESSOS OXIDATIVOS AVANÇADOS, São Paulo. Anais...

ASSOCIAÇÃO BRASILEIRA DE LIMPEZA PÚBLICA E RESÍDUOS ESPECIAIS (ABRELPE). (2017) Panorama dos resíduos sólidos no Brasil. São Paulo: ABRELPE. 124 p.

BARROS NETO, B.; SCARMINIO, J.S.; BURNS, R.R. (2001) Planejamento e otimização de experimentos. Campinas: Editora da Unicamp. 401 p.
BRASIL. (2012) Ministério do Meio Ambiente. Plano Nacional de Resíduos Sólidos. Brasília: Ministério do Meio Ambiente.

CASTILHOS JUNIOR, A. B. (org.). (2006) Gerenciamento de Resíduos Sólidos Urbanos com Ênfase na Proteção dos Corpos Dágua: Prevenção, Geração e Tratamento de Lixiviados de Aterros Sanitários. Programa de Pesquisa em Saneamento Básico (PROSAB). Edital 4. Rio de Janeiro: ABES. 494 p.

CHAOUKI, Z.; KHALIL, F.; IJJAALI, M.; VALDÉS, H.; RAFQAH, S.; SARAKHA, S.; ZAITAN, $H$. Use of combination of coagulation and adsorption process for the landfill leachate treatment from Casablanca city. Desalination and Water Treatment, v. 83, p. 262-271, 2017. http://dx.doi.org/10.5004/dwt.2017.20743

CHENG, G.;LI,Q.;SU,Z:SHENG,S.; FU,J.(2018)Preparation, optimization, and application of sustainable ceramsite substrate from coal fly ash/ waterworks sludge/oyster shell for phosphorus immobilization in constructed wetlands. Journal of Cleaner Production, v. 175, p. 572-581. http://dx.doi.org/10.1016/j.jclepro.2017.12.102

CHOU, Y.-C.; LO, S.-L.; KUO, J.; YEH, C.J. (2O13) A study on microwave oxidation of landfill leachate-Contributions of microwave-specific effects. Journal of Hazardous Materials, v. 246-247, p. 79-86. https:// doi.org/10.1016/j.jhazmat.2012.11.060 
EL-FADEL, M.; BOU-ZEID, E.; CHAHINE, W.; ALAYLI, B. (2002) Temporal variation of leachate quality from pre-sorted and baled municipal solid waste with high organic and moisture content. Waste Management, v. 22, n. 3, p. 269-282.

FOO, K.Y; HAMEED, B.H. (2009) An overview of landfill leachate treatment via activated carbon adsorption process. Journal of Hazardous Materials, v. 171, n. 1-3, p. 54-60.https://doi.org/10.1016/j. jhazmat.2009.06.038

HALIM, A.A.; AZIZ, H.A.; JOHARI, M.A.M.; ARIFFIN, K.S. (2O10) Comparison study of ammonia and COD adsorption on zeolite, activated carbon and composite materials in landfill leachate treatment. Desalination, v. 262, n1-3, p. 31-35. https://doi.org/10.1016/j. desal.2010.05.036

HAN, Z.Y.; LIU, D.; LI, Q.B. (2013) A removal mechanism for organics and nitrogen in treating leachate using a semi-aerobic aged refuse biofilter. Journal of Environmental Management, v. 114, p. 336-342. https://doi.org/10.1016/j.jenvman.2012.10.026

HSU, T.-C. (2009) Experimental assessment of adsorption of Cu2+ and $\mathrm{Ni2}+$ from aqueous solution by oyster shell powder. Journal of Hazardous Materials, v. 171, p. 995-1.000.

INTERNATIONAL UNION OF PURE AND APPLIED CHEMISTRY (IUPAC). (1985) Recommendations. Pure and Applied Chemistry, v. 57, n. 4, p. 603-619

KAWAHIGASHI, F; MENDES, M.B.; ASSUNÇÃO JÚNIOR, V.G.; GOMES, V.H.; FERNANDES, F.; HIROOKA, E.Y.; KURODA, E.K. (2O14) Pós-tratamento de lixiviado de aterro sanitário com carvão ativado. Engenharia Sanitária e Ambiental, v. 19, n. 3, p. 235-244. https:/l dx.doi.org/10.1590/S1413-41522014019000000652

KARGI, F.: PAMUKOGLU, M.Y. (2003) Simultaneous adsorption and biological treatment of pre-treated landfill leachate by fed-batch operation. Process Biochemistry, v. 38, n. 10, p. 1413-1420. http:// dx.doi.org/10.1016/SO032-9592(O3)00030-X

KURNIAWAN, T.A.; CHAN, G.Y.S.; LO, W.H.; BABEL, S. (2006) Comparisons of low-cost adsorbents for treating wastewaters laden with heavy metals. Science of the Total Environment, v. 366, n. 2-3, p. 409-426. https://doi.org/10.1016/i.scitotenv.2005.10.001

KWON, H.-B.; LEE, C.-W.; JUN, B.-S.; YUN, J-D.; WEON, S.Y.; KOOPMAN, B. (2004) Recycling waste oyster shells for eutrophication control. Resources, Conservation and Recycling, v. 41, n. 1, p. 75-82. http://doi. org/10.1016/..resconrec.2003.08.005

LI, W.; HU, T:; ZHOU, Q.; ZHANG, S.; LI, F. (2O10) Treatment of stabilized landfill leachate by the combined process of coagulation/flocculation and powder activated carbon adsorption. Desalination, v. 264, n. 1, p. 56-62. http://dx.doi. org/10.1016/j.desal.2010.07.004

LIM, J.E.; AHMAD, M.; USMAN, A.R.A.; LEE, S.S.; JEON, W.T.; OH, S.E.; YANG, J.E.; OK, Y.S. (2012) Effects of natural and calcined poultry waste on $\mathrm{Cd}, \mathrm{Pb}$ and $\mathrm{As}$ mobility in contaminated soil. Environmental Earth Sciences, v. 69, n. 1, p. 11-20. http://doi. org/10.1007/s12665-012-1929-z
LIN, L.; CHAN, G.Y.S.; JIANG, B.L.; LAN, C.Y. (2007) Use of ammoniacal nitrogen tolerant microalgae in landfill leachate treatment. Waste Management, v. 27, n. 10, p. 1376-1382. https://doi.org/10.1016/j. wasman.2006.09.001

LIU, X.; LI, X.-M.; YANG, Q.; YUE, X.; SHEN, T.T.; ZHENG, W.; LUO, K. SUN, Y.H.; ZENG, G.M. (2O12) Landfill leachate pretreatment by coagulation-flocculation process using iron-based coagulants: Optimization by response surface methodology. Chemical Engineering Journal, v. 200-202, p. 39-51. https://doi.org/10.1016/j. cej.2012.06.012

MARAÑÓN, E.; CASTRILLÓN, L.; FERNANDEZZNAVA, Y.; FERNÁNDEZ-MÉNDEZ, A.; FERNÁNDEZ-SÁNCHEZ, A. (2008) Coagulation-flocculation as a pretreatment process at a landfill leachate nitrification-denitrification plant. Journal of Hazardous Materials, v. 156, n. 1-3, p. 538-544. https://doi.org/10.1016/j. jhazmat.2007.12.084

MARTIN, C.D.; JOHNSON, K.D. (1995) The use of extended aeration and in-series surface-flow wetlands for landfill leachate treatment. Water Science \& Technology, v. 32, n. 2, p. 119-128. https://doi. org/10.2166/wst.1995.0133

MELLO, V.F.B.; ABREU, J.P.G.; FERREIRA, J.M.; JUCA, J.F.T.; MOTTA SOBRINHO, M.A. (2012) Variáveis no processo de coagulação / floculação/decantação de lixiviados de aterros sanitários urbanos. Ambi-Água, v. 7, n. 2, p. 88-100. http://dx.doi.org/10.4136/ ambi-agua.861

MORAVIA, W.G.; AMARAL, M.C.S.; LANGE, L.C. (2012) Evaluation of landfill leachate treatment by advanced oxidative process by Fenton's reagent combined with membrane separation system Waste Management, v. 33, n. 1, p. 89-101. https://doi.org/10.1016/j. wasman.2012.08.009

PALA, A.; ERDEN, G. (2004) Chemical pretreatment of landfill leachate discharged into municipal biological treatment systems Environmental Engineering Science, v. 21, n. 5, p. 549-557. https://doi. org/10.1089/ees.2004.21.549

Paulino, P.M.S. (2011) Avaliação do processo de coagulação/ floculação seguido de radiação gama para tratamento de lixiviados. 134f. Dissertação (Mestrado em Engenharia Civil) - Universidade Federal de Pernambuco, Recife.

RENOU, S.; GIVAUDAN, J.G.; POULAIN, S.; DIRASSOUYAN, F; MOULIN, P. (2008a). Landfill leachate treatment: Review and opportunity. Journal of Hazardous Materials, v. 150, n. 3, p. $468-493$. https://doi.org/10.1016/j.jhazmat.2007.09.077

RENOU, S.; POULAIN, S.; GIVAUDAN, J.G.; MOULIN, P. (2008b) Treatment process adapted to stabilized leachates: lime precipitation-prefiltration-reverse osmosis. Journal of Hazardous Materials, v. 313, n. 1-2, p. 9-22. http://dx.doi.org/10.1016/j. memsci.2007.11.023

RICHARDS, R.G.; MULLINS, B.J. (2013) Using microalgae for combined lipid production and heavy metal removal from leachate Ecological Modelling, v. 249, p. 59-67. http://dx.doi.org/10.1016/j. ecolmodel.2012.07.004 
ROBINSON, H.D.; GRANTHAM, G. (1988) The treatment of landfill leachates in on-site aerated lagoon plants: experience in Britain and Ireland. Water Research, v. 22, n. 6, p. 733-747. https://doi. org/10.1016/0043-1354(88)90184-4

ROCHA, E.M.R. (2005) Desempenho de um Sistema de Lagoas de Estabilização na Redução da Carga Orgânica do Percolado Gerado no Aterro da Muribeca (PE). 151f. Dissertação (Mestrado em Engenharia Civil) - Universidade Federal de Pernambuco, Recife.

ROCHEBROCHARD, S.; NAFFRECHOUX, E.; DROGUI, P.; MERCIER, G.; BLAIS, J.-F. (2013) Low frequency ultrasoundassisted leaching of sewage sludge for toxic metal removal, dewatering and fertilizing properties preservation. Ultrasonics Sonochemistry, v. 20, n. 1, p. 109-117. https://doi.org/10.1016/j. ultsonch.2012.08.001

RODRIGUES FILHO, G.M.; ROCHA, O.R.S.; MOTTA, M.A.; SILVA, V.L. (2008) Tratamento De Chorume Em Reator De Luz Artificial Branca Utilizando O Processo Foto Fenton Like. In: CONGRESSO BRASILEIRO DE ENGENHARIA QUÍMICA, 17., Recife. Anais...

SEO, D.C.; CHO, J.S.; LEE, H.J.; HEO, J.S. (2005) Phosphorus retention capacity of filter media for estimating the longevity of constructed wetland. Water Research, v. 39, n. 11, p. 2445-2457. https://doi. org/10.1016/j.watres.2005.04.032

SINGH, S.K.; TANG, W.Z. (2013) Statistical analysis of optimum Fenton oxidation conditions for landfill leachate treatment. Waste Management, v. 33, n. 1, p. 81-88. https://doi.org/10.1016/j. wasman.2012.08.005

SINGH, S.K.; TOWNSEND, T.G.; MAZYCK, D.; BOYER, T.H. (2O12) Equilibrium and intra-particle diffusion of stabilized landfill leachate onto micro- and meso-porous activated carbon. Water Research, v. 46, n. 2, p. 491-499. https://doi.org/10.1016/j.watres.2011.11.007

SONG, T.-H.; GAO, Y.J. (2013) Removal of Heavy Metals from Synthetic Landfill Leachate Using Oyster Shells Adsorbent.
Asian Journal of Chemistry, v. 25, n. 15, p. 8533-8536. http://dx.doi. org/10.14233/ajchem.2013.14828

TATSI, A.A.; ZOUBOULIS, I.; MATIS, K.A.; SAMARAS, P. (2003) Coagulation-flocculation pretreatment of sanitary landfill leachates. Chemosphere, v. 53, n. 7. p. 737-744. https://doi.org/10.1016/S00456535(03)00513-7

WANG, S.; WU, X.; WANG, Y.; LI, Q.; TAO, M. (2008) Removal of organic matter and ammonia nitrogen from landfill leachate by ultrasound. Ultrasonics Sonochemistry, v. 15, n. 6, p. 933-937. https:// doi.org/10.1016/j.ultsonch.2008.04.006

WANG, X.J.; CHEN, S.L.; GU, X.Y.; WANG, K.Y. (2009) Pilot study on the advanced treatment of landfill leachate using a combined coagulation, Fenton oxidation and biological aerated filter process. Waste Management, v. 29, n. 4, p. 1354-1358. https://doi.org/10.1016/j. wasman.2008.10.006

XU, Z.Y.; ZENG, G.M.; YANG, Z.H.; XIAO, Y.; CAO, M.; SUN, H.S.; JI, L.L.; CHEN, Y. (2O1O) Biological treatment of landfill leachate with the integration of partial nitrification, anaerobic ammonium oxidation and heterotrophic denitrification. Bioresource Technology, v. 101, n. 1. p. 79-86. https://doi.org/10.1016/j.biortech.2009.07.082

XUAN, Z.; CHANG, N.B.; WANIELISTA, M.; HOSSAIN, F. (2O10) Laboratory-Scale Characterization of a Green Sorption Medium for On-Site Sewage Treatment and Disposal to Improve Nutrient Removal. Environmental Engineering Science, v. 27, n. 4, p. 301-312. https://doi.org/10.1089/ees.2009.0256

ZHANG, C.; ZENG, G.; YUAN, L.; YU, J.; LI, J.; HUANG, G.; XI, B.; LIU, $\mathrm{H}$. (2007) Aerobic degradation of bisphenol A by Achromobacter xylosoxidans strain B-16 isolated from compost leachate of municipal solid waste. Chemosphere, v. 68, n. 1, p. 181-190. https:// doi.org/10.1016/j.chemosphere.2006.12.012

ZHANG, H.; CHOI, H.J.; HUANG, C.P. (2005) Optimization of Fenton process for the treatment of landfill leachate. Journal of Hazardous Materials, v. 125, n. 1-3, p. 166-174. https://doi.org/10.1016/j. jhazmat.2005.05.025 